\title{
Emerging Technologies that will impact on the UK food system
}

Area of research interest: Emerging challenges and opportunities

Project status: Completed

Conducted by: University of Cambridge

Date published: 4 June 2021

DOI: https://doi.org/10.46756/sci.fsa.srf852

Rapid technological innovation is reshaping the UK food system in many ways. We need to stay abreast of these changes and develop regulatory responses to ensure novel technologies do not compromise food safety and public health.

Six technology fields were identified and their implications for industry, consumers, food safety and the regulatory framework explored. These fields are:

- Food Production and Processing (indoor farming, 3D food printing, food side and byproduct use, novel non-thermal processing, and novel pesticides)

- Novel Sources of Protein, such as insects (for human consumption, and animal feedstock)

- Synthetic Biology (including lab-grown meat and proteins)

- Genomics Applications along the value chain (for food safety applications, and personal "nutrigenomics")

- Novel Packaging (active, smart, biodegradable, edible, and reusable solutions) Digital Technologies in the food sector (supporting analysis, decision making and traceability)

Research report

PDF

View Emerging technologies that will impact on the UK food system as PDF(Open in a new window) (1.27 MB) 